\title{
Equivariant collaring, tubular neighbourhood and gluing theorems for proper Lie group actions
}

\author{
MARJA KANKAANRINTA
}

\begin{abstract}
The purpose of this paper is to prove equivariant versions of some basic theorems in differential topology for proper Lie group actions. In particular, we study how to extend equivariant isotopies and then apply these results to obtain equivariant smoothing and gluing theorems. We also study equivariant collars and tubular neighbourhoods. When possible, we follow the ideas in the well-known book of M W Hirsch. When necessary, we use results from the differential topology of Hilbert spaces.
\end{abstract}

$57 \mathrm{~S} 20$

\section{Introduction}

The aim of this paper is to prove equivariant versions of some basic theorems in differential topology, for proper actions of not necessarily compact Lie groups. If a Lie group $G$ acts properly and smoothly on a smooth manifold $M$, then its action resembles a smooth action of a compact Lie group. The reason for this is that the slice theorem, which is one of the most important basic results in the theory of compact transformation groups, also holds for proper actions. According to the slice theorem, if $x \in M$, then a $G$-invariant neighbourhood of $x$ is $G$-diffeomorphic to the twisted product $G \times{ }_{G_{x}} \mathrm{~N}_{x}$, where $G_{x}$ denotes the isotropy subgroup at $x$ and $\mathrm{N}_{x}=\mathrm{T}_{x} M / \mathrm{T}_{x}(G x)$ is the normal space to the orbit $G x$ at $x$. There are different ways to formulate the slice theorem. The first variant is due to J L Koszul [7, p 139]. The version we refer to is due to R S Palais [13, Proposition 2.2.2].

Our main results concern equivariant isotopies. Isotopies are basic constructions in differential topology, and isotopy extension results have proved to be especially useful. For example, in order to extend an embedding it is sufficient to prove that it is isotopic to an extendable embedding. In this paper, we study how to extend equivariant isotopies and apply these results to obtain an equivariant smoothing theorem (Theorem 9.4). We also prove a uniqueness theorem for the equivariant diffeomorphism type of the adjunction space $M \cup_{f} N$, where $M$ and $N$ are proper smooth $G$-manifolds which have been equivariantly glued together along their boundaries (Theorem 10.1). 
For the sake of background, let us mention another reason why equivariant isotopies are important. It is natural to ask whether it is possible to classify $G$-spaces over a given space. To prove a classifying result of this type, one needs to be able to lift homotopies or isotopies from orbit spaces. For continuous actions, R S Palais' covering homotopy theorem [12] is a fundamental lifting result for homotopies of maps between orbit spaces. Palais' theorem was extended in a highly nontrivial way by G W Schwarz to the smooth case [15]. Both results are stated for compact group actions, but they also hold for proper actions of not necessarily compact Lie groups.

Some of the results in this paper concerning equivariant collars and tubular neighbourhoods may be known to the experts. We include them here in order to provide detailed proofs for the literature. We hope that this paper will be of use in further study and understanding of differential topology of smooth $G$-manifolds.

The paper is organized as follows: We first recall some basic properties of proper smooth $G$-manifolds in Section 2. In Section 3 we prove an equivariant collaring theorem (Theorem 3.5), ie we show that the boundary of a proper smooth $G$-manifold $M$ has an equivariant collar on $M$. We continue by proving an equivariant tubular neighbourhood theorem for manifolds without boundary (Theorem 4.4). Section 6 shows some technical results concerning extensions of smooth equivariant maps. In Section 7, we study equivariant collars and tubular neighbourhoods of neat submanifolds. The results of Section 6 and Section 7 are needed in Section 9, where we prove an equivariant smoothing theorem (Theorem 9.4). For that we also need results about extending equivariant isotopies (Section 8). Finally, Section 10 deals with equivariant gluing.

Most of the corresponding nonequivariant results can be found in a book of Hirsch [3]. Roughly, our results in Section 3 and Section 7 correspond to those in Section 4.6 in [3], and our results in Section 4 correspond to those in Section 4.5 in [3]. Moreover, the results in Section 8 and Section 9 are equivariant versions of the results in [3, Section 8.1] and our results in Section 10 correspond to those in [3, Section 8.2].

In several of his proofs (for example the collaring theorem [3, Theorem 4.6.1]) Hirsch uses a globalization theorem [3, Theorem 2.2.11] to obtain maps with required properties. This approach does not generalize well to the equivariant setting, which is one reason why some of our proofs differ from those of Hirsch. Unlike Hirsch we restrict our attention to closed submanifolds. That's why using the exponential map suits well to our purposes. For example, we use the standard method, based on the use of the exponential map, to construct $G$-invariant tubular neighbourhoods.

Another reason why some of the proofs in [3] need to be modified in the equivariant case is that smooth manifolds can always be embedded in Euclidean spaces, while 
the corresponding equivariant results do not always hold even if the acting Lie group is compact. Fortunately, proper smooth $G$-manifolds can be embedded in Hilbert $G$-spaces. An equivariant tubular neighbourhood theorem for finite-dimensional closed submanifolds of a Hilbert $G$-space is proved in Section 5.

\section{Proper smooth $G$-manifolds}

Let $X$ be a Hausdorff space and let $G$ be a Lie group acting continuously on $X$. Let $A$ and $B$ be subsets of $X$. We denote by $G(A, B)$ the subset $\{g \in G \mid g A \cap B \neq \varnothing\}$ of $G$. We call a subset $A$ of $X$ relatively compact if its closure $\bar{A}$ is compact. The interior of $A$ is denoted by $\dot{A}$. Let $f: X \rightarrow \mathbb{R}$ be a continuous map. We denote the support of $f$, ie the closure of the set $\{x \in X \mid f(x) \neq 0\}$, by $\operatorname{supp}(f)$.

Definition 2.1 The action of $G$ on $X$ is called proper, if for every two points $x$ and $y$ in $X$ there are neighbourhoods $U$ and $V$ of $x$ and $y$, respectively, such that $G(U, V)$ is relatively compact.

The action of $G$ on $X$ is proper, if and only if the map $G \times X \rightarrow X \times X$ taking $(g, x)$ to $(g x, x)$ is proper.

Let a Lie group $G$ act smoothly on a smooth (ie $\mathrm{C}^{\infty}$ ) manifold $M$. If the action map $G \times M \rightarrow M$ is smooth, we call $M$ a smooth $G$-manifold. If the action is also proper, we call $M$ a proper smooth $G$-manifold.

All the manifolds are assumed to be finite-dimensional and to have only countably many connected components. They are allowed to have a nonempty boundary unless the contrary is mentioned.

We recall the notion of a smooth slice:

Definition 2.2 Let $G$ be a Lie group and let $M$ be a proper smooth $G$-manifold. Let $x \in M$ and let $G_{x}$ denote the isotropy subgroup at $x$. A smooth submanifold $S$ of $M$ is called a smooth slice at $x$ if $x \in S, G S$ is open in $M$ and there exists a smooth $G$-equivariant map $f: G S \rightarrow G / G_{x}$ such that $f^{-1}\left(e G_{x}\right)=S$.

By Proposition 2.2.2 in [13], there exists a smooth slice at each point of a proper smooth $G$-manifold.

Let $F$ be a subset of $M$. If every point $x \in M$ has a neighbourhood $U$ such that $G(U, F)$ is relatively compact, we call $F$ small. 
Definition 2.3 Let $G$ be a Lie group and let $M$ be a proper smooth $G$-manifold. If $F$ is small and $G F=M$, we call $F$ a fundamental set for $G$ in $M$. If, in addition, $F$ is closed in $M$, we call it a closed fundamental set. We call a closed fundamental set $F$ in $M$ fat, if $G \dot{F}=M$.

By Lemma 3.6 in Illman and Kankaanrinta [4], a proper smooth $G$-manifold always has a fat closed fundamental set.

We call a Euclidean space on which $G$ acts linearly a linear $G$-space.

Lemma 2.4 Let $G$ be a Lie group and let $M$ be a proper smooth $G$-manifold. Let $f: M \rightarrow \mathbb{V}$ be a smooth map into a linear $G$-space $\mathbb{V}$. Assume the support of $f$ is small. Then

$$
\operatorname{Av}(f): M \rightarrow \mathbb{V}, \quad x \mapsto \int_{G} g f\left(g^{-1} x\right) d g,
$$

where the integral is the left Haar integral over $G$, is a smooth $G$-equivariant map.

Proof It follows from Proposition 1.2.6 in Palais [13], that $\operatorname{Av}(f)$ is a well-defined continuous $G$-equivariant map. Since the support of $f$ is small, the smoothness follows just like in the case where $G$ is compact. For example, one can apply the proof of Theorem 0.3.3 in Bredon [1] inductively, to prove the smoothness.

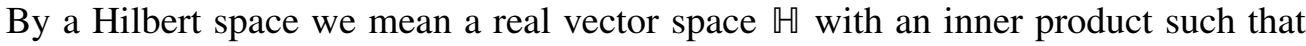
relative to the metric induced by the inner product, $\mathbb{H}$ is a complete metric space. We denote the group of orthogonal linear transformations of $\mathbb{U}$ by $\mathrm{O}(\mathbb{M})$.

Definition 2.5 Let $G$ be a Lie group and let $\mathbb{H}$ be a Hilbert space. If there exists a representation $\varrho: G \rightarrow \mathrm{O}(\mathbb{H})$, such that the action $G \times \mathbb{H} \rightarrow \mathbb{H},(g, v) \mapsto \varrho(g) v$, is continuous, we call $\mathbb{H}$ a Hilbert $G$-space.

Let $G$ be a Lie group and let $M$ be a proper smooth $G$-manifold. By Theorem 0.1 in Kankaanrinta [6], there exists a smooth $G$-equivariant embedding of $M$ as a closed smooth submanifold of some Hilbert $G$-space $\mathbb{H}$. The result in [6] is stated for manifolds without boundary but that assumption is not used anywhere in the proof, so the result also holds for manifolds with boundary. It follows [6, Theorem 0.2] that every proper smooth $G$-manifold (with or without boundary) admits a complete smooth $G$-invariant Riemannian metric. 


\section{Proof of the collaring theorem}

Several different proofs for the nonequivariant collaring theorem are known (see for example Theorems 4.6.1 and 6.2.1 in Hirsch [3]). The proof of Theorem 4.6.1 in [3] can be adapted to the equivariant case. Hence we continue by proving Proposition 3.1, Proposition 3.2 and Proposition 3.4 whose nonequivariant versions are being used in that proof.

Proposition 3.1 Let $G$ be a Lie group and let $M$ be a proper smooth $G$-manifold with boundary $\partial M$. Then there exist an open $G$-invariant neighbourhood $W$ of $\partial M$ in $M$ and a smooth $G$-equivariant retraction $r: W \rightarrow \partial M$.

Proof Exactly the same as the proof of (a smooth version of ) Proposition 1.4 in Illman and Kankaanrinta [5], but by using only inward pointing normal vectors.

Proposition 3.2 Let $G$ be a Lie group and let $M$ be a proper smooth $G$-manifold with boundary $\partial M$. Then there exist an open $G$-invariant neighbourhood $U$ of $\partial M$ in $M$ and a smooth $G$-invariant map $f: U \rightarrow[0, \infty)$ having 0 as a regular value and taking $\partial M$ to 0 .

Proof Let exp and TM denote the exponential map and the tangent bundle of $M$, respectively. Let $\mathrm{T}(\partial M)^{\perp}$ denote the orthogonal complement of $\mathrm{T}(\partial M)$ in $\left.(\mathrm{T} M)\right|_{\partial M}$ with respect to a smooth $G$-invariant Riemannian metric $\langle$,$\rangle of M$. Moreover, let $\mathrm{T}(\partial M)_{i}^{\perp}$ denote the inward pointing normal vectors in $\mathrm{T}(\partial M)^{\perp}$. Then $\partial M$ has an open $G$-invariant neighbourhood $U$ in $M$ and the zero section of $\mathrm{T}(\partial M)_{i}^{\perp}$ has an open $G$-invariant neighbourhood $V$ such that the restriction $\exp \mid: V \rightarrow U$ is a smooth $G$-equivariant diffeomorphism. The map

$$
f: U \rightarrow[0, \infty), \quad x \mapsto\left\langle\left.\exp \right|^{-1}(x),\left.\exp \right|^{-1}(x)\right\rangle^{\frac{1}{2}}
$$

has the required properties.

Lemma 3.3 Let $G$ be a Lie group and let $M$ be a proper smooth $G$-manifold without boundary. Assume $G$ acts trivially on $[0, \infty)$ and diagonally on $M \times[0, \infty)$. Let $V$ be a $G$-invariant neighbourhood of $M \times\{0\}$ in $M \times[0, \infty)$. Then there exists a smooth $G$-invariant map $\theta: M \rightarrow(0, \infty)$ such that $(x, y) \in V$, for every $0 \leq y \leq \theta(x)$.

Proof Let $E$ be a fat closed fundamental set in $M$. Let $\alpha: M \rightarrow[0,1]$ be a smooth map whose support lies in $\dot{E}$. We can assume that $\alpha$ is not identically zero on any orbit of $M$. Let $\beta: M \rightarrow(0, \infty)$ be a smooth map such that $(x, y) \in V$, for every 
$0 \leq y \leq \beta(x)$. Let $g \in G$. Since $V$ is $G$-invariant, it follows that $(g x, y) \in V$, for every $0 \leq y \leq \beta(x)$. Let $\theta$ be the map

$$
\theta: M \rightarrow(0, \infty), \quad x \mapsto \frac{\int_{G} \alpha\left(g^{-1} x\right) \beta\left(g^{-1} x\right) d g}{\int_{G} \alpha\left(g^{-1} x\right) d g} .
$$

By Lemma 2.4, $\theta$ is a smooth $G$-invariant map. Let $x \in M$. Then $\theta(x) \leq \beta(z)$, for some $z \in \operatorname{supp}\left(\left.\alpha\right|_{G x}\right)$. It follows that $\theta$ satisfies the required properties.

Proposition 3.4 Let $G$ be a Lie group and let $M$ be a proper smooth $G$-manifold without boundary. Let $G$ act trivially on $[0, \infty)$ and diagonally on $M \times[0, \infty)$. Let $V$ be a $G$-invariant neighbourhood of $M \times\{0\}$ in $M \times[0, \infty)$. Then there exists a smooth $G$-equivariant embedding $\varphi: M \times[0, \infty) \rightarrow V$ such that $\varphi(x, 0)=(x, 0)$, for every $x \in M$.

Proof Let $\theta: M \rightarrow(0, \infty)$ be as in Lemma 3.3 and let $e: \mathbb{R} \rightarrow \mathbb{R}$ be the exponential map. Then

$$
\varphi: M \times[0, \infty) \rightarrow V, \quad(x, y) \mapsto\left(x, \theta(x)\left(1-e^{-y}\right)\right),
$$

is the required embedding.

Assume $G$ is a Lie group and $M$ is a proper smooth $G$-manifold with boundary $\partial M$. Let $G$ act trivially on the interval $[0, \infty)$. By an equivariant collar of $\partial M$ on $M$ we mean a smooth $G$-equivariant embedding

$$
f: \partial M \times[0, \infty) \rightarrow M
$$

such that $f(x, 0)=x$, for every $x \in \partial M$. An equivariant collaring theorem holds for proper smooth actions:

Theorem 3.5 Let $G$ be a Lie group and let $M$ be a proper smooth $G$-manifold with boundary $\partial M$. Then $\partial M$ has an equivariant collar on $M$.

Proof Let the maps $r$ and $f$ be as in Proposition 3.1 and Proposition 3.2, respectively, and let

$$
h: W \cap U \rightarrow \partial M \times[0, \infty), \quad x \mapsto(r(x), f(x)) .
$$

Then $h$ is a smooth $G$-equivariant map and $h(x)=(x, 0)$, for every $x \in \partial M$. The restriction of $h$ to $\partial M$ is a $G$-homeomorphism onto $\partial M \times\{0\}$. Since $h$ is submersive (and therefore also immersive) at the boundary points, it is a local diffeomorphism on some neighbourhood of the boundary $\partial M$. It now follows from Lemma 1.3 in [5], that $\partial M$ has an open $G$-invariant neighbourhood $V$ in $W \cap U$ such that $h \mid: V \rightarrow h(V)$ is a $G$-equivariant diffeomorphism and $h(V)$ is open in $\partial M \times[0, \infty)$. By Proposition 3.4, 
there exists a smooth $G$-equivariant embedding $\varphi: \partial M \times[0, \infty) \rightarrow h(V)$ which fixes $\partial M \times\{0\}$. Then the composed map $h^{-1} \circ \varphi$ is an equivariant collar of $\partial M$ on $M$.

Let $\mathrm{T}_{x} M$ denote the tangent space of $M$ at $x$. Then the normal space at $x$ to the orbit $G x$ is $\mathrm{N}_{x}=\mathrm{T}_{x} M / \mathrm{T}_{x}(G x)$. The linear slice theorem for manifolds without boundary says that if $x \in M \backslash \partial M$, then a $G$-invariant neighbourhood of $x$ is $G$-equivariantly diffeomorphic to the twisted product $G \times{ }_{G_{x}} \mathrm{~N}_{x}$. This fact and the equivariant collaring theorem now imply Corollary 3.6, which is a linear slice theorem for manifolds with boundary:

Corollary 3.6 Let $G$ be a Lie group and let $M$ be a proper smooth $G$-manifold with boundary $\partial M$. If $x \in \partial M$, then a $G$-invariant neighbourhood of $x$ is $G$-equivariantly diffeomorphic to $\left(G \times_{G_{x}} \overline{\mathrm{N}}_{x}\right) \times[0, \infty)$, where $\overline{\mathrm{N}}_{x}=\mathrm{T}_{x}(\partial M) / \mathrm{T}_{x}(G x)$.

Corollary 3.6 says that every proper smooth action of a Lie group on a smooth manifold with boundary is locally linear (called locally smooth in Bredon [1]).

The action of a Lie group $G$ on a topological manifold $M$ with boundary is called locally linear, if every $x \in M \backslash \partial M$ has a neighbourhood $G$-equivariantly homeomorphic to $G \times_{G_{x}} \mathbb{V}_{x}$ and every $x \in \partial M$ has a neighbourhood which is $G$-equivariantly homeomorphic to $\left(G \times_{G_{x}} \mathbb{V}_{x}\right) \times[0, \infty)$, where $\mathbb{V}_{x}$ is a linear $G_{x}$-space. For compact $G$, a topological version of the equivariant collaring theorem is known [1, Theorem $\mathrm{V}$ 1.5]. This theorem is proved for locally linear actions. The corresponding nonequivariant result was proved by M Brown [2]. Since the covering homotopy theorem [12] of R S Palais holds for proper actions, it is easy to verify that the proof of Bredon's theorem also works for proper locally linear actions. Thus we obtain a topological version of the equivariant collaring theorem:

Theorem 3.7 Let $G$ be a Lie group and let $M$ be a topological manifold with boundary $\partial M$. Assume $G$ acts properly and locally linearly on $M$. Then there exists a $G$-equivariant homeomorphism $h$ of $\partial M \times[0, \infty)$ onto a neighbourhood of $\partial M$ in $M$ with $h(x, 0)=x$, for every $x \in \partial M$.

\section{$4 G$-invariant tubular neighbourhoods}

Let $G$ be a Lie group acting smoothly and properly on a smooth manifold $M$. Let $\xi=(p, E, M)$ be a smooth vector bundle over $M$. Assume $G$ acts smoothly on $E$ and in such a way that the action is linear on the fibers. Moreover, assume the projection $p: E \rightarrow M$ is equivariant. We then call $\xi$ a smooth $G$-vector bundle over $M$. 
Definition 4.1 Let $G$ be a Lie group and let $M$ be a proper smooth $G$-manifold. Let $N$ be a closed smooth $G$-invariant submanifold of $M$. By a $G$-invariant tubular neighbourhood of $N$ in $M$ we mean a pair $(\varphi, \xi)$, where $\xi=(p, E, N)$ is a smooth $G$-vector bundle over $N$ and $\varphi: E \rightarrow M$ is a smooth $G$-equivariant embedding onto some open neighbourhood of $N$ in $M$, such that the restriction of $\varphi$ to the zero section (identified with $N$ ) of $\xi$ is the inclusion of $N$ in $M$.

Associated to $\varphi(E)$ is a particular smooth $G$-equivariant retraction $r: \varphi(E) \rightarrow N$. We often refer to $E, \varphi(E)$ or $r: \varphi(E) \rightarrow N$ as a $G$-invariant tubular neighbourhood of $N$ in $M$.

The next lemma is proved for actions of compact Lie groups in [1, Theorem VI 2.1].

Lemma 4.2 Let $G$ be a Lie group and let $M$ be a proper smooth $G$-manifold. Let $\xi=(p, E, M)$ be a smooth $G$-vector bundle over $M$. Then there exists a smooth positive definite $G$-invariant inner product on $\xi$.

Proof Let $E \oplus E$ denote the Whitney sum and let $p: E \oplus E \rightarrow M$ denote the projection. Let $F$ be a fat closed fundamental set in $M$. Then $p^{-1}(F)$ is a fat closed fundamental set in $E \oplus E$. Let $\alpha: M \rightarrow[0, \infty)$ be a smooth map with support in $\dot{F}$ and such that $\alpha$ is not identically zero on any orbit. Let $\langle$,$\rangle be a smooth positive$ definite inner product on $\xi$. (Such an inner product exists; see eg [1, Theorem VI 2.1].) We define a new inner product $\{$,$\} on \xi$ by putting

$$
\{v, w\}_{x}=\int_{G} \alpha\left(g^{-1} x\right)\left\langle g^{-1} v, g^{-1} w\right\rangle_{g^{-1} x} d g .
$$

By Lemma 2.4, $\{$,$\} is smooth and G$-invariant. Clearly, it is positive definite.

By a $G$-invariant partial tubular neighbourhood of a closed smooth $G$-invariant submanifold $N$ of a proper smooth $G$-manifold $M$ we mean a triple $(f, \xi, U)$ where $\xi=(p, E, N)$ is a smooth $G$-vector bundle over $N, U$ is a $G$-invariant neighbourhood of the zero section in $E$ and $f: U \rightarrow M$ is a smooth $G$-equivariant embedding such that the restriction $f \mid N=\operatorname{id}_{N}$ and $f(U)$ is open in $M$.

The following proposition shows that a $G$-invariant partial tubular neighbourhood always contains a $G$-invariant tubular neighbourhood. The proof is just as in the nonequivariant case [3, p 109].

Proposition 4.3 Let $G$ be a Lie group and let $M$ be a proper smooth $G$-manifold. Let $N$ be a closed smooth $G$-invariant submanifold of $M$. Assume $(f, \xi, U)$ is a $G$-invariant partial tubular neighbourhood of $N$ in $M$. Then there exists a $G$-invariant 
tubular neighbourhood $(s, \xi)$ of $N$ in $M$ such that $s$ equals $f$ in a neighbourhood of $N$.

Proof By Lemma $4.2, \xi$ has a smooth positive definite $G$-invariant inner product $\langle$,$\rangle . Let \|\|$ be the norm $\langle$,$\rangle induces on the fibers in E$. Let $\varrho: N \rightarrow(0, \infty)$ be a smooth $G$-invariant map such that if $y \in E_{x}$ and $\|y\| \leq \varrho(x)$, then $y \in U$. Let $\lambda:[0, \infty) \rightarrow[0,1)$ be a diffeomorphism which equals the identity near 0 . We define

$$
h: E \rightarrow E, \quad y \mapsto\left\{\begin{aligned}
\varrho(p(y)) \lambda\left(\frac{\|y\|}{\varrho(p(y))}\right)\|y\|^{-1} y, & \text { if }\|y\| \neq 0 \\
y, & \text { otherwise. }
\end{aligned}\right.
$$

Then $h$ is a $G$-equivariant map, $h(E) \subset U$ and $h$ is the identity near the zero section. It is left for the reader to verify that $h$ is a smooth embedding. Let $s=f \circ h$. Then $(s, \xi)$ is a $G$-invariant tubular neighbourhood of $N$ with the required properties.

Theorem 4.4 and Theorem 4.6 are equivariant versions of Theorems 4.5.2 and 4.5.3 in [3], respectively. However, notice that Hirsch does not require $N$ to be closed in $M$. Equivariant versions for compact $G$ can be found in [1, Theorems VI 2.2 and VI 2.6].

Theorem 4.4 Let $G$ be a Lie group and let $M$ be a proper smooth $G$-manifold. Let $N$ be a closed smooth $G$-invariant submanifold of $M$. Assume $\partial M=\partial N=\varnothing$. Then $N$ has a $G$-invariant tubular neighbourhood in $M$.

Proof Like the proof of Proposition 1.4 in [5].

Let $G$ be a Lie group and let $M$ and $N$ be proper smooth $G$-manifolds. By a $G-$ equivariant isotopy from $M$ to $N$ we mean a smooth map $F: N \times I \rightarrow M$ such that for each $t \in I$ the map

$$
F_{t}: N \rightarrow M, x \mapsto F(x, t),
$$

is a smooth $G$-equivariant embedding. We call the embeddings $F_{0}$ and $F_{1} G-$ equivariantly isotopic (or $G$-isotopic). If $A \subset M$ is such that $F_{t}(x)=F_{0}(x)$, for all $(x, t) \in A \times I$, then we call $F$ a $G$-equivariant rel $A$ isotopy. In the case when $N=M$ and each $F_{t}$ is a diffeomorphism, we call $F$ a $G$-equivariant diffeotopy (or a $G$-diffeotopy). Notice that we do not require $F_{0}$ to be the identity map of $M$ as Hirsch does when he defines a diffeotopy [3, p 178]. For an isotopy $F$ we define a map

$$
\widehat{F}: N \times I \rightarrow M \times I, \quad(x, t) \mapsto(F(x, t), t) .
$$

Definition 4.5 Let $\left(f_{i}, \xi_{i}=\left(p_{i}, E_{i}, N\right)\right), i=0,1$, be $G$-invariant tubular neighbourhoods of $N$ in $M$. By a $G$-equivariant isotopy of $G$-invariant tubular neighbourhoods from $\left(f_{0}, \xi_{0}\right)$ to $\left(f_{1}, \xi_{1}\right)$ we mean a $G$-equivariant rel $N$ isotopy $F: E_{0} \times I \rightarrow M$ such that 
- $F_{0}=f_{0}$,

- $F_{1}\left(E_{0}\right)=f_{1}\left(E_{1}\right)$,

- $f_{1}^{-1} \circ F_{1}: E_{0} \rightarrow E_{1}$ is a vector bundle isomorphism $\xi_{0} \rightarrow \xi_{1}$,

- $\hat{F}\left(E_{0} \times I\right)$ is open in $M \times I$.

Notice that the last condition is always true, if $\partial M=\varnothing$. Notice also that $F_{1}$ and $f_{1}$ necessarily define the same retraction $F_{1}\left(E_{0}\right) \rightarrow N$.

Theorem 4.6 Let $G$ be a Lie group and let $M$ be a proper smooth $G$-manifold. Let $N$ be a closed smooth $G$-invariant submanifold of $M$. Assume $\partial M=\partial N=\varnothing$. Then any two $G$-invariant tubular neighbourhoods of $N$ in $M$ are $G$-equivariantly isotopic.

Proof The proof is like that of Theorem 4.5.3 in [3]. The map $H$ in Hirsch's proof becomes $G$-equivariant when $\left(f_{0}, \xi_{0}\right)$ and $\left(f_{1}, \xi_{1}\right)$ are $G$-invariant. Using Lemma 4.2, it is also possible to make Hirsch's maps $h$ and $G G$-equivariant.

\section{Tubular neighbourhoods in Hilbert $G$-spaces}

In this section we prove an equivariant tubular neighbourhood theorem (Theorem 5.1) for finite-dimensional closed submanifolds in Hilbert $G$-spaces. The result is needed later, in Section 6 and Section 7.

For elements of infinite-dimensional differential geometry we refer to Lang [8]. Let $\mathbb{W}$ be a Hilbert space. The exponential map of $\mathbb{U}$ defined by the trivial spray over $\mathbb{U}$

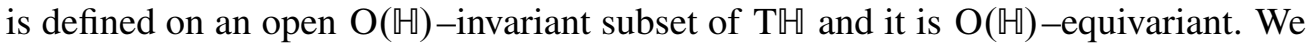
obtain:

Theorem 5.1 Let $G$ be a Lie group and let $M$ be a proper smooth $G$-manifold with $\partial M=\varnothing$. Let $f: M \rightarrow \mathbb{H}$ be a closed smooth $G$-equivariant embedding in a Hilbert $G$-space $\mathbb{H}$. Then $f(M)$ has a $G$-invariant tubular neighbourhood in $\mathbb{U}$.

Proof The claim can be proved like Theorem IV.5.1 in [8], by using the exponential map of $\mathbb{~}$. Notice that we do not need partitions of unity, since in Lang's proof they are only used to construct a global spray. The trivial spray certainly is global.

Remark 5.2 Let $W$ be a $G$-invariant tubular neighbourhood of $f(M)$. Since $G$ acts properly on $f(M)$ and there exists an equivariant retraction $r: W \rightarrow f(M)$, it follows that $G$ acts properly also on $W$. 


\section{Extending smooth $G$-equivariant maps}

In this section we show how to extend certain kind of smooth equivariant maps equivariantly. Corollary 6.3 will be applied in Section 9.

Lemma 6.1 Let $G$ be a Lie group and let $M$ and $N$ be proper smooth $G$-manifolds without boundary. Let $G$ act trivially on $\mathbb{R}$ and diagonally on $M \times \mathbb{R}$ and $N \times \mathbb{R}$. Assume $f=\left(f_{1}, f_{2}\right): M \times(-\infty, 0] \rightarrow N \times(-\infty, 0]$ is a smooth $G$-equivariant map such that $f_{2}(x, 0)=0$, for every $x \in M$. Then, for every $x \in M$, there exists a slice $S_{x}$ at $x$ such that the restriction $f \mid\left(G S_{x} \times(-\infty, 0]\right)$ can be extended to a smooth $G$-equivariant map $f_{x}: G S_{x} \times\left(-\infty, a_{x}\right) \rightarrow N \times \mathbb{R}$, for some $a_{x}>0$.

Proof The map $f$ can be extended to a smooth (nonequivariant) map $h: U \rightarrow N \times \mathbb{R}$, where $U$ is a sufficiently small neighbourhood of $M \times(-\infty, 0]$ in $M \times \mathbb{R}$. Let $x \in M$ and let $S \subset N$ be a smooth slice at $f_{1}(x, 0)$. Then $S \times \mathbb{R}$ is a smooth slice at $f(x, 0)$. Let $r: W \rightarrow S$ be a $G_{x}$-invariant tubular neighbourhood of $S$ in $G S$. We can assume that $S$ and $W$ are relatively compact. Thus $W$ has only finitely many orbit types and, consequently, there exists a closed smooth $G_{x}$-equivariant embedding $e: W \rightarrow \mathbb{R}^{n}(\varrho)$, where $\mathbb{R}^{n}(\varrho)$ is a linear $G_{x}$-space on which $G_{x}$ acts via some homomorphism $\varrho: G_{x} \rightarrow \mathrm{O}(n)$ (see Mostow [10] or Palais [11]).

Now, $h^{-1}(W \times \mathbb{R})$ is open in $M \times \mathbb{R}$. Let $S_{x} \subset M$ be a smooth slice at $x$, and let $J_{x}$ be an open interval containing 0 . By choosing $S_{x}$ and $J_{x}$ to be sufficiently small, we can assume that $S_{x} \times J_{x} \subset h^{-1}(W \times \mathbb{R})$.

Let $r^{\prime}: W^{\prime} \rightarrow e(W)$ be a $G_{x}$-invariant tubular neighbourhood of $e(W)$ in $\mathbb{R}^{n}(\varrho)$. Let id: $\mathbb{R} \rightarrow \mathbb{R}$ be the identity map. Then

$$
H_{x}=(e \times \mathrm{id}) \circ h \mid: S_{x} \times J_{x} \rightarrow \mathbb{R}^{n}(\varrho) \times \mathbb{R}
$$

is a smooth map. Since $G_{x}$ is compact, it follows that

$$
\operatorname{Av}\left(H_{x}\right): S_{x} \times J_{x} \rightarrow \mathbb{R}^{n}(\varrho) \times \mathbb{R}, \quad(y, t) \mapsto \int_{G_{x}} g H_{x}\left(g^{-1} y, t\right) d g,
$$

is a smooth $G_{x}$-equivariant map. By shrinking $S_{x}$ and $J_{x}$, if necessary, we can assume that $\operatorname{Av}\left(H_{x}\right)\left(S_{x} \times J_{x}\right) \subset W^{\prime} \times \mathbb{R}$. Then

$$
\tilde{H}_{x}=\left(e^{-1} \times \mathrm{id}\right) \circ\left(r^{\prime} \times \mathrm{id}\right) \circ \operatorname{Av}\left(H_{x}\right): S_{x} \times J_{x} \rightarrow N \times \mathbb{R}
$$

is a smooth $G_{x}$-equivariant map. Since $S_{x} \times J_{x}$ is a smooth slice at $(x, 0)$, the map

$$
h_{x}: G S_{x} \times J_{x} \rightarrow N \times \mathbb{R}, \quad(g y, t) \mapsto g \tilde{H}_{x}(y, t),
$$


is smooth and $G$-equivariant. Defining

$$
f_{x}:\left(G S_{x} \times(-\infty, 0]\right) \cup\left(G S_{x} \times J_{x}\right) \rightarrow N \times \mathbb{R}
$$

by $f_{x}(y, t)=f(y, t)$ if $(y, t) \in G S_{x} \times(-\infty, 0]$ and $f_{x}(y, t)=h_{x}(y, t)$ if $(y, t) \in$ $G S_{x} \times J_{x}$, yields a smooth $G$-equivariant map with the desired properties.

Theorem 6.2 Let $G, M, N$ and $f$ be as in Lemma 6.1. Then there exist a $G-$ invariant neighbourhood $V$ of $M \times(-\infty, 0]$ in $M \times \mathbb{R}$ and a smooth $G$-equivariant map $F: V \rightarrow N \times \mathbb{R}$ extending $f$.

Proof We begin by covering $M \times\{0\}$ locally finitely by tubes $G S_{x}$, where $S_{x} \subset M \times \mathbb{R}$ is a smooth slice at $(x, 0), x \in M$. It is possible to do this in such a way that the family $\left\{G S_{x}\right\}$ can be divided to finitely many subfamilies $\left\{G S_{x_{i}}\right\}_{i \in \alpha_{j}}, 1 \leq j \leq n$, for some $n \in \mathbb{N}$, such that for all $j, G S_{x_{i}} \cap G S_{x_{k}}=\varnothing$, if $i, k \in \alpha_{j}$ and $i \neq k$ [12, Theorem 1.8.2]. We denote

$$
O_{j}=(M \times(-\infty, 0)) \cup\left(\bigcup_{i \in \alpha_{j}} G S_{x_{i}}\right),
$$

for all $j$. By Lemma 6.1, $f$ has a smooth $G$-equivariant extension to $G S_{x_{i}}$, for every $i \in \alpha_{j}$. These extensions define a smooth $G$-equivariant extension of $f$,

$$
F_{j}: O_{j} \rightarrow N \times \mathbb{R}
$$

Let $\left\{f_{j}\right\}_{j=1}^{n}$ be a smooth $G$-invariant partition of unity subordinate to $\left\{O_{j}\right\}_{j=1}^{n}$ [14, Theorem 4.2.4 (4)]. Let $e: N \rightarrow \mathbb{H}$ be a closed smooth $G$-equivariant embedding into a Hilbert $G$-space $\mathbb{H}$ and let $r: W \rightarrow e(N)$ be a smooth $G$-invariant tubular neighbourhood of $e(N)$ in $\mathbb{H}$. Let id denote the identity map of $\mathbb{R}$. For all $j$, define the map $\bar{F}_{j}$ by

$$
\bar{F}_{j}: \bigcup_{j=1}^{n} O_{j} \rightarrow \mathbb{H} \times \mathbb{R}, \quad(y, t) \mapsto\left\{\begin{aligned}
f_{j}(y, t)(e \times \mathrm{id})\left(F_{j}(y, t)\right), & \text { if }(y, t) \in O_{j} \\
0, & \text { otherwise. }
\end{aligned}\right.
$$

We can assume that $\Sigma_{j} \bar{F}_{j}(y, t) \in W \times \mathbb{R}$, for all $(y, t)$. Let

$$
F: \bigcup_{j=1}^{n} O_{j} \rightarrow N \times \mathbb{R}, \quad(y, t) \mapsto\left(e^{-1} \times \mathrm{id}\right)(r \times \mathrm{id})\left(\sum_{j=1}^{n} \bar{F}_{j}(y, t)\right) .
$$

Then $F$ is a smooth $G$-equivariant map extending $f$.

Theorem 6.2 and Lemma 1.3 in [5] imply the following: 
Corollary 6.3 Let $G$ be a Lie group and let $M$ be a proper smooth $G$-manifold. Let $G$ act trivially on $\mathbb{R}$ and diagonally on $M \times \mathbb{R}$. Let $f: M \times(-\infty, 0] \rightarrow M \times(-\infty, 0]$ be a smooth $G$-equivariant diffeomorphism. Then there exist a $G$-invariant neighbourhood $V$ of $M \times(-\infty, 0]$ in $M \times \mathbb{R}$ and a smooth $G$-equivariant diffeomorphism $F: V \rightarrow F(V)$ extending $f$.

\section{$7 \quad G$-invariant collars and tubular neighbourhoods of closed neat submanifolds}

Let $G$ be a Lie group and let $M$ be a proper smooth $G$-manifold. Let $N$ be a smooth $G$-invariant submanifold of $M$. Following the terminology in [3], we call $N$ a neat submanifold of $M$, if $\partial N=N \cap \partial M$ and if $N$ is covered by charts $(\varphi, U)$ of $M$ such that $N \cap U=\varphi^{-1}\left(\mathbb{R}^{m}\right)$ where $m=\operatorname{dim}(N)$. Thus $N$ is neat if and only if $\partial N=N \cap \partial M$ and for every $x \in \partial N, \mathrm{~T}_{x} N$ is not a subspace of $\mathrm{T}_{x}(\partial M)$.

Theorems 7.2, 7.3 and 7.4 are equivariant versions of Theorems 4.6.2, 4.6.3 and 4.6.4 in [3], respectively. Again, notice that in 4.6.3 and 4.6.4, Hirsch does not require the submanifolds to be closed.

Lemma 7.1 Let $G$ be a Lie group and let $M$ be a proper smooth $G$-manifold. Let $N$ be a closed smooth neat $G$-invariant submanifold of $M$. Then there exist a $G-$ invariant neighbourhood $U_{1}$ of $\partial N$ in $M$ and a smooth $G$-equivariant retraction $r_{1}: U_{1} \rightarrow U_{1} \cap \partial M$ such that $r_{1}(x) \in \partial N$ for every $x \in U_{1} \cap N$.

Proof By the equivariant collaring theorem, $\partial M$ has a neighbourhood in $M$ which is $G$-equivariantly diffeomorphic to $\partial M \times[0, \infty)$. Let $s: U \rightarrow \partial N$ be a $G$-invariant tubular neighbourhood of $\partial N$ in $\partial M$. Let $e: U \rightarrow \mathbb{H}$ be a closed smooth $G$-equivariant embedding in some Hilbert $G$-space $\mathbb{U}$ and let $r^{\prime}: V \rightarrow e(U)$ be a $G$-invariant tubular neighbourhood of $e(U)$ in $\mathbb{H}$. Moreover, let id denote the identity map of $[0, \infty)$ and let $e_{0}=e \times$ id: $U \times[0, \infty) \rightarrow \mathbb{W} \times[0, \infty)$.

Fix a smooth $G$-invariant Riemannian metric on $N$. Let $\mathrm{T}(\partial N)_{i}^{\perp}$ denote the inward pointing normal vectors in the orthogonal complement $\mathrm{T}(\partial N)^{\perp}$ of $\mathrm{T}(\partial N)$ in $\left.(\mathrm{T} N)\right|_{\partial N}$. Let exp denote the restriction of the exponential map of $N$ to T $(\partial N)_{i}^{\perp}$. Let $1_{s(v)}^{\perp}$ denote the inward pointing unit vector in $\mathrm{T}_{s(v)} N / \mathrm{T}_{s(v)}(\partial N)$. Since $\mathrm{T}(\partial N)_{i}^{\perp}$ is trivial [3, Theorem 4.4.2], the map $f: O \rightarrow U \times[0, \infty)$ defined by

$$
(v, t) \mapsto e_{0}^{-1} \circ\left(r^{\prime} \times \mathrm{id}\right) \circ\left(e_{0}(v)+e_{0}\left(\exp _{s(v)}\left(t \cdot 1_{s(v)}^{\perp}\right)\right)-e_{0}(s(v))\right)
$$

is well-defined on some $G$-invariant neighbourhood $O$ of $\partial N$ in $U \times[0, \infty)$. Then $f$ is a smooth $G$-equivariant map and its restriction to some $G$-invariant neighbourhood 
$W$ of $\partial N$ is a diffeomorphism onto $f(W)$. Moreover, we may assume that the image $f(\partial N \times[0, \infty) \cap W)=N \cap f(W)$. Denote the inverse of $f \mid W$ by $f^{-1}$. Let pr: $U \times[0, \infty) \rightarrow U$ be the projection. Then pro $f^{-1}: f(W) \rightarrow U$ is a smooth $G$-equivariant retraction such that $\operatorname{pr} \circ f^{-1}(x) \in \partial N$ for every $x \in N \cap f(W)$.

Theorem 7.2 Let $G$ be a Lie group and let $M$ be a proper smooth $G$-manifold. Let $N$ be a closed smooth neat $G$-invariant submanifold of $M$. Then $\partial M$ has a $G$-equivariant collar which restricts to a $G$-equivariant collar of $\partial N$ in $N$.

Proof The proof is similar to the proof of Theorem 3.5, if we manage to choose the maps $r$ and $\varphi$ in that proof in such a way that $r: W \rightarrow \partial M$ maps $N \cap W$ onto $\partial N$ and that $\varphi(x, t) \in N \times[0, \infty)$ for every $(x, t) \in N \times[0, \infty)$. By choosing $V$, as in Theorem 3.5, to be sufficiently small, we can then assume that the restricted map $h \mid: V \cap N \rightarrow h(V) \cap(\partial N \times[0, \infty))$ is a diffeomorphism. That $\varphi$ has the required property, follows immediately from its definition, see Proposition 3.4.

It remains to construct a smooth $G$-equivariant retraction $r$ from an open $G$-invariant neighbourhood $W$ of $\partial M$ to $\partial M$ such that $r(x) \in \partial N$ for every $x \in N \cap W$. First, let $U_{1}$ and $r_{1}$ be as in Lemma 7.1. Next, let $U_{2}$ be an open $G$-invariant subset in $M$ such that $\partial M \subset U_{1} \cup U_{2}$ and $N \cap U_{2}=\varnothing$. Choosing $U_{2}$ to be sufficiently small and using the collaring theorem, we obtain a smooth $G$-equivariant retraction $r_{2}: U_{2} \rightarrow \partial M \cap U_{2}$. Let $\left\{f_{1}, f_{2}\right\}$ be a smooth $G$-invariant partition of unity such that $\operatorname{supp}\left(f_{1}\right) \subset U_{1}$ and $\operatorname{supp}\left(f_{2}\right) \subset U_{2}$. Let $e: \partial M \rightarrow \mathbb{W}$ be a closed smooth $G$-equivariant embedding into a Hilbert $G$-space $\mathbb{U}$. For $i=1,2$, we define the map

$$
\bar{r}_{i}: U_{1} \cup U_{2} \rightarrow \mathbb{H}, x \mapsto\left\{\begin{aligned}
f_{i}(x) e\left(r_{i}(x)\right), & \text { if } x \in U_{i} \\
0, & \text { otherwise. }
\end{aligned}\right.
$$

By Theorem 5.1, there is a smooth $G$-invariant tubular neighbourhood $\bar{r}: O \rightarrow e(\partial M)$ of $e(\partial M)$ in $\mathbb{H}$. When $U_{1}$ and $U_{2}$ are sufficiently small, ie such that $\bar{r}_{1}(x)+\bar{r}_{2}(x) \in O$, for every $x \in U_{1} \cup U_{2}$, the map

$$
r: U_{1} \cup U_{2} \rightarrow \partial M, x \mapsto e^{-1} \circ \bar{r}\left(\bar{r}_{1}(x)+\bar{r}_{2}(x)\right),
$$

is well-defined. Clearly, $r$ then is a smooth $G$-equivariant retraction taking points of $N$ to $\partial N$.

In the following theorem we construct tubular neighbourhoods for neat submanifolds. It is easy to see that the standard method based on the use of the exponential map does not work here. Therefore, we follow the idea of Hirsch, although some of our technical details are slightly different due to the fact that we need Hilbert spaces in situations where Hirsch uses Euclidean ones. 
Theorem 7.3 Let $G$ be a Lie group and let $M$ be a proper smooth $G$-manifold. Let $N$ be a closed smooth neat $G$-invariant submanifold of $M$. Then $N$ has a $G$-invariant tubular neighbourhood in $M$.

Proof By Theorem 7.2, there exist a $G$-invariant neighbourhood $V_{1}$ of $\partial M$ in $M$ and a $G$-equivariant diffeomorphism

$$
\varphi:\left(V_{1}, \partial M\right) \approx_{G}(\partial M \times[0,1), \partial M \times\{0\})
$$

such that

$$
\varphi \mid: V_{1} \cap N \approx_{G} \partial N \times[0,1) .
$$

Let $V_{2}$ be an open $G$-invariant subset in $M$ such that $M \backslash V_{1} \subset V_{2}$. Let $h_{1}, h_{2}: M \rightarrow \mathbb{R}$ be a smooth $G$-invariant partition of unity with $\operatorname{supp}\left(h_{1}\right) \subset V_{1}$ and $\operatorname{supp}\left(h_{2}\right) \subset V_{2}$.

Let $e_{1}: \partial M \rightarrow \mathbb{H}_{1}$ and $e_{2}: V_{2} \rightarrow \mathbb{H}_{2}$ be closed smooth $G$-equivariant embeddings in some Hilbert $G$-spaces $\mathbb{H}_{1}$ and $\mathbb{H}_{2}$, respectively. Let $\lambda:[0,1) \rightarrow[0, \infty)$ be a diffeomorphism and let id denote the identity map of $\partial M$. Then

$$
\widehat{e}_{1}: \partial M \times[0, \infty) \rightarrow \mathbb{H}_{1} \times[0, \infty),(x, t) \mapsto\left(e_{1}(x), t\right),
$$

is a closed smooth $G$-equivariant embedding. Moreover, the maps

$$
\tilde{e}_{1}=\widehat{e}_{1} \circ(\mathrm{id} \times \lambda) \circ \varphi: V_{1} \rightarrow \mathbb{H}_{1} \times[0, \infty)
$$

and

$$
\tilde{e}_{2}: V_{2} \rightarrow \mathbb{H}_{2} \times[0, \infty), x \mapsto\left(e_{2}(x), 1\right)
$$

are closed smooth $G$-equivariant embeddings. For $i=1,2$, define

$$
f_{i}: M \rightarrow \mathbb{H}_{i} \times[0, \infty), x \mapsto\left\{\begin{aligned}
h_{i}(x) \widetilde{e}_{i}(x), & \text { if } x \in V_{i} \\
0, & \text { otherwise }
\end{aligned}\right.
$$

Then the map

$$
F: M \rightarrow \mathbb{R} \times \mathbb{R} \times \mathbb{H}_{2} \times \mathbb{H}_{1} \times \mathbb{R}=\mathbb{U} \times \mathbb{R}, \quad x \mapsto\left(h_{1}(x), h_{2}(x), f_{1}(x)+f_{2}(x)\right),
$$

is a closed smooth $G$-equivariant embedding in the Hilbert $G$-space $\mathbb{H} \times \mathbb{R}$, where $\mathbb{H}=\mathbb{R} \times \mathbb{R} \times \mathbb{H}_{2} \times \mathbb{H}_{1}$. Thus $F$ embeds $M$ in $\mathbb{H} \times[0, \infty) \subset \mathbb{H} \times \mathbb{R}$ in such a way that every vector of $\mathbb{H} \times \mathbb{R}$ which is normal to $F\left(V_{1}\right)$ at a point of $F(\partial M)$ or normal to $F\left(V_{1} \cap N\right)$ at a point of $F(\partial N)$, is in $\mathbb{H}$.

The image $F(M)$ is a closed smooth neat $G$-invariant submanifold of the $G$-space $\mathbb{H} \times[0, \infty)$. For $x \in F(M)$, let $\mathrm{T}_{x}(F(M))^{\perp}$ denote the orthogonal complement of $\mathrm{T}_{x}(F(M))$ in $\mathbb{U} \times \mathbb{R}$. Then $\mathrm{T}(F(M))^{\perp} \subset F(M) \times \mathbb{W} \times \mathbb{R}$. Thus

$$
f: \mathrm{T}(F(M))^{\perp} \rightarrow \mathbb{H} \times \mathbb{R}, \quad(x, y) \mapsto x+y,
$$

where $x \in F(M)$ and $y \in \mathrm{T}_{x}(F(M))^{\perp}$, is a smooth $G$-equivariant map whose restriction to the zero section is the identity. Moreover, $d f_{x}$ is a continuous bijection 
at points of $F(M)$. By the inverse function theorem [8, Theorem 1.5.2], $f$ is a local diffeomorphism on $F(M)$. It follows that $f \mid: W \rightarrow f(W) \subset \mathbb{} \times[0, \infty)$ is a $G$-equivariant diffeomorphism for some $G$-invariant neighbourhood $W$ of $F(M)$ in $\mathrm{T}(F(M))^{\perp}$ and $f(W)$ is open in $\mathbb{H} \times[0, \infty)$. Hence we obtain a smooth $G$-equivariant retraction $r: f(W) \rightarrow F(M)$. Notice that we can't apply Lemma 1.3 in [5] here, since that lemma is stated for locally compact spaces only. Instead, the shrinking can be done like, for example, in the end of the proof of Theorem IV.5.1 in [8].

We give $M$ the $G$-invariant Riemannian metric induced from $\mathbb{M} \times[0, \infty)$. Let $v=(p, E, N)$ be the normal bundle of $N$ in $M$. Thus

$$
v \subset(\mathrm{T} M)_{N} \subset(\mathrm{T}(\mathbb{H} \times[0, \infty)))_{N}=F(N) \times \mathbb{H} \times \mathbb{R},
$$

ie each fibre $v_{x}$ is contained in $\{F(x)\} \times \mathbb{U} \times \mathbb{R}$.

Let $x \in N$. We define $U_{x}=\left\{(F(x), y) \in v_{x} \mid F(x)+y \in f(W)\right\}$. Then $U=\bigcup_{x \in N} U_{x}$ is an open $G$-invariant subset of $E$ and the map $s: U \rightarrow M$ sending $(F(x), y)$ to $F^{-1} \circ r(F(x)+y)$ provides a $G$-invariant partial tubular neighbourhood for $N$ in $M$. Applying Proposition 4.3 proves the theorem.

Theorem 7.4 Let $G$ be a Lie group and let $M$ be a proper smooth $G$-manifold. Let $N$ be a closed smooth neat $G$-invariant submanifold of $M$. Then every $G$-invariant tubular neighbourhood of $\partial N$ in $\partial M$ is the intersection with $\partial M$ of a $G$-invariant tubular neighbourhood of $N$ in $M$.

Proof We first consider the special case $M=W \times I, N=U \times I$, where $U$ is a closed smooth $G$-invariant submanifold of $W$ and $\partial U=\partial W=\varnothing$. Then

$$
\text { and } \quad \begin{aligned}
\partial M & =W \times\{0\} \cup W \times\{1\} \\
\partial N & =U \times\{0\} \cup U \times\{1\} .
\end{aligned}
$$

A $G$-invariant tubular neighbourhood of $\partial N$ in $\partial M$ is a pair of $G$-invariant tubular neighbourhoods of $U$ in $W$. Let these be $E_{0}$ and $E_{1}$. By Theorem 4.6, there is a $G$-equivariant isotopy of tubular neighbourhoods from $E_{0}$ to $E_{1}$. We denote this isotopy by $F: E_{0} \times I \rightarrow W$. The corresponding embedding

$$
\widehat{F}: E_{0} \times I \rightarrow W \times I=M, \quad(y, t) \mapsto(F(y, t), t),
$$

defines a $G$-invariant tubular neighbourhood for $U \times I=N$ in $M$, and this tubular neighbourhood restricts to $E_{0}$ and $E_{1}$ in $\partial M$.

We next consider the general case. By Theorem 7.2, $\partial M$ has a $G$-equivariant collar in $M$ which restricts to a $G$-equivariant collar of $\partial N$ in $N$. We identify $\partial M \times[0, \infty)$ 
with a $G$-invariant neighbourhood $O_{M}$ of $\partial M$ in $M$, so that $\partial N \times[0, \infty)$ corresponds to a $G$-invariant neighbourhood $O_{N}$ of $\partial N$ in $N$. Let

$$
\begin{aligned}
M^{\prime} & =\partial M \times[0,1], & & N^{\prime}=\partial N \times[0,1] \\
M^{\prime \prime} & =M \backslash(\partial M \times[0,1)), & N^{\prime \prime} & =N \backslash(\partial N \times[0,1)) .
\end{aligned}
$$

Then $M=M^{\prime} \cup M^{\prime \prime}, M^{\prime} \cap M^{\prime \prime}=\partial M \times\{1\}, N=N^{\prime} \cup N^{\prime \prime}, N^{\prime} \cap N^{\prime \prime}=\partial N \times\{1\}$. Both $M^{\prime \prime}$ and $N^{\prime \prime}$ are smooth manifolds with boundary and $N^{\prime \prime}$ is a neat submanifold of $M^{\prime \prime}$.

Let $E_{0}$ be a $G$-invariant tubular neighbourhood of $\partial N \times\{0\}$ in $\partial M \times\{0\}$. By Theorem 7.3, there is a $G$-invariant tubular neighbourhood $E$ of $N^{\prime \prime}$ in $M^{\prime \prime}$. Let $E_{1}=E \cap(\partial M \times\{1\}) \subset \partial M \times\{1\}$. Then $E_{0}$ and $E_{1}$ form a $G$-invariant tubular neighbourhood for $\partial N \times\{0,1\}$ in $\partial M \times\{0,1\}$. By the special case, we can extend $E_{0} \cup E_{1}$ to a $G$-invariant tubular neighbourhood $E^{\prime}$ of $N^{\prime}$ in $M^{\prime}$.

Let $\tau:[0,1] \rightarrow[0,1]$ be a smooth increasing surjection collapsing a neighbourhood of 1 to 1 , and let id be the identity map of $E_{0}$. Taking the composed map $F \circ(\mathrm{id} \times \tau)$, we can assume that the isotopy from $E_{0}$ to $E_{1}$, which defines $E^{\prime}$, is constant near 1 .

Let $s:[1, \infty) \rightarrow[1, \infty)$ be a smooth surjection which collapses a neighbourhood of 1 to 1 and increases on $\left[1 \frac{1}{2}, \infty\right)$. We can assume that $s$ equals 1 on $\left[1,1 \frac{1}{2}\right]$ and that $s$ equals the identity map on $[2, \infty)$. Write $N^{\prime \prime}=(\partial N \times[1, \infty)) \cup\left(N \backslash O_{N}\right)$. Let

$$
f=\left(\operatorname{id}_{\partial N} \times s\right) \cup \operatorname{id}_{\left(N \backslash O_{N}\right)}:(\partial N \times[1, \infty)) \cup\left(N \backslash O_{N}\right) \rightarrow(\partial N \times[1, \infty)) \cup\left(N \backslash O_{N}\right) .
$$

The pullback of $E$ by $f$ defines a smooth $G$-vector bundle $E^{\prime \prime}$ over $N^{\prime \prime}$. The $G$-vector bundles $E^{\prime}$ and $E^{\prime \prime}$ fit together smoothly at $\partial N \times\{1\}$, forming a smooth $G$-vector bundle $E^{\prime} \cup E^{\prime \prime}$ over $N$. Let $p: E^{\prime} \cup E^{\prime \prime} \rightarrow N$ be the projection.

Let $\varphi: E \rightarrow M^{\prime \prime}$ and $\varphi^{\prime}: E^{\prime} \rightarrow M^{\prime}$ denote the smooth $G$-equivariant embeddings defining the tubular neighbourhoods of $N^{\prime \prime}$ in $M^{\prime \prime}$ and $N^{\prime}$ in $M^{\prime}$, respectively. If $y \in E$ and $\varphi(y) \in \partial M \times[1, \infty)$, we write $\varphi(y)=\left(\varphi_{1}(y), \varphi_{2}(y)\right) \in \partial M \times[1, \infty)$. Now consider the function $\bar{\varphi}: E^{\prime} \cup E^{\prime \prime} \rightarrow M$ defined by:

$$
y \mapsto\left\{\begin{aligned}
\varphi^{\prime}(y), & \text { if } p(y) \in N^{\prime} \\
\left(\varphi_{1}(y), \varphi_{2}(y)+t-s(t)\right), & \text { if } p(y)=(x, t) \in \partial N \times(1,3] \\
\varphi(y), & \text { if } p(y) \in N^{\prime \prime} \backslash(\partial N \times[1,3])
\end{aligned}\right.
$$

This is a well-defined smooth $G$-equivariant map. Clearly, it's restriction to the zero section is a diffeomorphism onto $N$, and it is immersive at each point of the zero section. Thus the restriction $\bar{\varphi} \mid U$, where $U$ is some open $G$-invariant neighbourhood of the zero section, defines a $G$-invariant partial tubular neighbourhood of $N$ in $M$. We can assume that $E^{\prime} \backslash p^{-1}(\partial N \times\{1\}) \subset U$. 
The restriction $\bar{\varphi} \mid: U \backslash p^{-1}(\partial N \times\{0\}) \rightarrow M \backslash \partial M$ defines a $G$-invariant partial tubular neighbourhood for $N \backslash \partial N$ in $M \backslash \partial M$. Choosing the map $\varrho: N \backslash \partial N \rightarrow(0, \infty)$, as in Proposition 4.3, in such a way that for $(x, t) \in \partial N \times(0,1), \varrho(x, t)$ only depends on $t$ and $\varrho(x, t) \rightarrow \infty$ when $t \rightarrow 0$, and applying Proposition 4.3, yields a $G$-invariant tubular neighbourhood of $N \backslash \partial N$ in $M \backslash \partial M$ which fits together smoothly with $E_{0}$. Thus the obtained $G$-invariant tubular neighbourhood of $N$ in $M$ extends $E_{0}$.

\section{Extending equivariant isotopies}

In this section we investigate equivariant isotopies. The results are equivariant versions of the corresponding theorems in Hirsch [3, Section 8].

Let $G$ be a Lie group and let $M$ be a proper smooth $G$-manifold. Let $X: M \rightarrow \mathrm{T} M$ be a smooth vector field. If $X_{g x}\left(f \circ g^{-1}\right)=X_{x}(f)$, for every $x \in M, g \in G$ and for every smooth real valued map $f$ defined on a neighbourhood of $x$ in $M$, we call $X$ an invariant vector field. If $X$ is an invariant vector field and $\sigma: I \rightarrow M$ is an integral curve of $X$ at $x \in M$, then $g \circ \sigma$ is an integral curve of $X$ at $g x \in M$.

By a time-dependent vector field on $M$ we mean a smooth map $Y: M \times I \rightarrow \mathrm{T} M$ such that $Y_{(x, t)}=Y(x, t) \in \mathrm{T}_{x} M$, for every $x \in M$. If $\partial M \neq \varnothing$, we also require that $Y(\partial M \times I) \subset \mathrm{T}(\partial M)$. The vector field $Y$ is called invariant, if the vector field $Y_{(, t)}: M \rightarrow \mathrm{T} M$ is invariant, for every $t \in I$. We say that a vector field $Y$ has bounded velocity, if $M$ has a complete smooth Riemannian metric $\langle$,$\rangle such that there$ exists a constant $K>0$ for which $\langle Y(x, t), Y(x, t)\rangle<K$, for every $(x, t) \in M \times I$.

Theorem 8.1 and Theorem 8.2 are equivariant versions of Theorems 8.1.1 and 8.1.2 in [3], respectively.

Theorem 8.1 Let $G$ be a Lie group and let $M$ be a proper smooth $G$-manifold. Let $Y$ be a time-dependent invariant vector field on $M$ having bounded velocity. Then there is a unique $G$-equivariant diffeotopy $F: M \times I \rightarrow M$ such that

$$
\frac{\partial F}{\partial t}(x, t)=Y(F(x, t), t) .
$$

Moreover, $F_{0}=\mathrm{id}_{M}$.

Proof Let $X: M \times I \rightarrow \mathrm{T}(M \times I)$ be the vector field $X(x, t)=(Y(x, t), 1)$. By Theorem 8.1.1 in [3], $Y$ generates a diffeotopy $F$. This diffeotopy is constructed in such a way that, for each $x \in M$, the map $I \rightarrow M \times I, t \mapsto(F(x, t), t)$, is an integral curve of $X$ at $(x, 0)$. Since $Y$ is invariant, it follows that $g F(x, t)=F(g x, t)$, ie $F$ is a $G$-equivariant diffeotopy. 
Let $Y: M \times I \rightarrow \mathrm{T} M$ be a time-dependent invariant vector field. By the support $\operatorname{supp}(Y) \subset M$ of $Y$ we mean the closure of $\{x \in M \mid Y(x, t) \neq 0$ for some $t \in I\}$. If $\operatorname{supp}(Y) / G$ is compact, then $Y$ has bounded velocity with respect to any complete smooth $G$-invariant Riemannian metric. As in the nonequivariant case, Theorem 8.1 implies:

Theorem 8.2 Let $G$ be a Lie group and let $M$ be a proper smooth $G$-manifold. A time-dependent invariant vector field $Y: M \times I \rightarrow \mathrm{T} M$ such that $\operatorname{supp}(Y) / G$ is compact generates a $G$-equivariant isotopy. In particular, if $G$ is compact, then every time-dependent invariant vector field on a compact smooth $G$-manifold generates a $G$-equivariant isotopy.

Let $N$ be a submanifold of $M$. An isotopy $F: N \times I \rightarrow M$ is said to have bounded velocity if $M$ has a complete smooth Riemannian metric such that the tangent vectors to the curves $t \mapsto F(x, t)$ have bounded lengths. We call the closure of the set $\{x \in N \mid F(x, t) \neq F(x, 0)$ for some $t \in I\}$ the support $\operatorname{supp}(F)$ of the isotopy $F: N \times I \rightarrow M$.

We next prove equivariant isotopy extension theorems, Theorem 8.3 and Theorem 8.6. These results are equivariant versions of Theorems 8.1.7 and 8.1.6 in [3], respectively.

Theorem 8.3 Let $G$ be a Lie group and let $M$ be a proper smooth $G$-manifold. Let $A$ be a closed $G$-invariant subset of $M$ and let $U$ be an open $G$-invariant neighbourhood of $A$ in $M$. Let $F: U \times I \rightarrow M$ be a $G$-equivariant isotopy of $U$ having bounded velocity, such that $\widehat{F}(U \times I)$ is open in $M \times I$ and $F_{0}$ is the canonical inclusion. Then there is a $G$-equivariant diffeotopy $\widetilde{F}$ of $M$ having bounded velocity, such that $\widetilde{F}$ agrees with $F$ on a neighbourhood of $A \times I, \operatorname{supp}(\widetilde{F}) \subset F(U \times I)$ and $\widetilde{F}_{0}=\operatorname{id}_{M}$.

Proof The tangent vectors to the curves

$$
\widehat{F}_{x}: x \times I \rightarrow M \times I, \quad(x, t) \mapsto(F(x, t), t),
$$

for $x \in U$, define a vector field $Y$ on $\hat{F}(U \times I)$, where $Y_{(F(x, t), t)}=\left(\frac{\partial F}{\partial t}(x, t), 1\right)$. The horizontal part of $Y$ is a vector field $X: \hat{F}(U \times I) \rightarrow \mathrm{T} M,(y, t) \mapsto X_{(y, t)} \in \mathrm{T}_{y} M$. The vector field $X$ is time-dependent and has bounded velocity. As $\hat{F}(U \times I)$ is an open $G$-invariant subset of $M \times I$ and since $\widehat{F}(A \times I) \subset \widehat{F}(U \times I)$ is a closed $G-$ invariant subset of $M \times I$, there exists a smooth $G$-invariant map $f: M \times I \rightarrow[0,1]$ such that $f(y, t)=1$ on a $G$-invariant neighbourhood $V$ of $\widehat{F}(A \times I)$ in $\widehat{F}(U \times I)$ and $\operatorname{supp}(f) \subset \widehat{F}(U \times I)$. The vector field

$$
\tilde{X}: M \times I \rightarrow \mathrm{T} M, \quad(y, t) \mapsto\left\{\begin{array}{cl}
f(y, t) X_{(y, t)}, & \text { if }(y, t) \in \hat{F}(U \times I) \\
0, & \text { otherwise }
\end{array}\right.
$$


is time-dependent and invariant. Moreover, $\tilde{X}$ agrees with $X$ on $V$.

Since $X$ has bounded velocity, $\tilde{X}$ also has bounded velocity. By Theorem $8.1, \tilde{X}$ generates a $G$-equivariant diffeotopy $\widetilde{F}: M \times I \rightarrow M$ where

$$
\frac{\partial \tilde{F}}{\partial t}(x, t)=\tilde{X}(\tilde{F}(x, t), t) \quad \text { and } \quad \tilde{F}_{0}=\operatorname{id}_{M} .
$$

Clearly, $\operatorname{supp}(\widetilde{F}) \subset F(U \times I)$. Since $\tilde{X}$ has bounded velocity, $\widetilde{F}$ does too. If $(x, t) \in \widehat{F}^{-1}(V)$, then

$$
\tilde{X}(F(x, t), t)=X(F(x, t), t)=\frac{\partial F}{\partial t}(x, t),
$$

and the uniqueness of the solutions implies that $\widetilde{F}$ agrees with $F$ on the $G$-invariant neighbourhood $\widehat{F}^{-1}(V)$ of $A \times I$.

Let $M$ be a connected smooth manifold and let $d$ be a metric on $M$ induced by a complete smooth Riemannian metric. In the proof of the following lemma we will use the well-known fact that every bounded subset of $M$ is relatively compact. Thus a subset of $M$ is compact if and only if it is closed and bounded.

Lemma 8.4 Let $G$ be a Lie group and let $M$ and $N$ be proper smooth $G$-manifolds, with $\partial M=\varnothing=\partial N$. Assume $F: N \times I \rightarrow M$ is a $G$-equivariant isotopy with bounded velocity and such that the map $F_{0}$ is a closed embedding. Then $\widehat{F}(N \times I)$ is a closed smooth neat $G$-invariant submanifold of $M \times I$.

Proof Clearly, $\hat{F}$ is a smooth injective $G$-equivariant immersion. Since neatness is also obvious, it remains to show that $\hat{F}$ is a closed map.

We first assume that $N$ has only finitely many connected components. Without loss of generality we may assume that $N$ and $M$ are connected. Let $d$ and $d^{\prime}$ be complete metrics on $N$ and $M$, respectively, induced by complete smooth Riemannian metrics $\langle$,$\rangle and \langle,\rangle^{\prime}$. Assume $F$ has bounded velocity with respect to $\langle,\rangle^{\prime}$.

Let $A$ be a closed subset of $N \times I$ and let $\left(x_{n}, t_{n}\right)$ be a point in $A$, for every $n \in \mathbb{N}$. Assume $\hat{F}\left(x_{n}, t_{n}\right)=\left(F\left(x_{n}, t_{n}\right), t_{n}\right) \rightarrow(y, t) \in M \times I$. Assume first that $\left(x_{n}\right)$ has no convergent subsequence. Let $z \in N$. We can now assume that $d\left(x_{n}, z\right) \rightarrow \infty$. Since $F_{0}$ is a proper map, it follows that also $d^{\prime}\left(F\left(x_{n}, 0\right), F(z, 0)\right) \rightarrow \infty$. It follows that $d^{\prime}\left(F\left(x_{n}, 0\right), y\right) \rightarrow \infty$. Since $F$ has bounded velocity, the paths $F\left(\left\{x_{n}\right\} \times I\right)$ have bounded lengths. This yields a contradiction with the fact that $F\left(x_{n}, t_{n}\right) \rightarrow y$. Thus we may assume that a subsequence of $\left(x_{n}, t_{n}\right)$ converges to a point $(x, t) \in A$, and it follows that $(y, t)=\widehat{F}(x, t) \in \widehat{F}(A)$. Thus $\widehat{F}(A)$ is closed in $M \times I$. 
Assume then that $N$ is allowed to have countably many connected components. Passing to a subsequence, if necessary, we may assume that each $x_{n}$ is in a different connected component of $N$. Then $\left(F\left(x_{n}, 0\right)\right)$ has no convergent subsequence. Again, this contradicts the assumption that $F\left(x_{n}, t_{n}\right) \rightarrow y$. It follows that $\widehat{F}(A)$ is closed in $M \times I$ and $\hat{F}$ is a closed map.

Notice that in Lemma 8.5 and in Theorem 8.6, $M \times I$ strictly speaking is a manifold with corners (see Mather [9]), if $\partial M \neq \varnothing$.

Lemma 8.5 Let $G$ be a Lie group and let $M$ be a proper smooth $G$-manifold. Let $N$ be a closed smooth $G$-invariant submanifold of $M$ with $\partial N=\varnothing$. Assume $F: N \times I \rightarrow M$ is a $G$-equivariant isotopy with bounded velocity such that either $F(N \times I) \subset \partial M$ or $F(N \times I) \subset M \backslash \partial M$. Assume $F_{0}$ is the canonical inclusion. Then $\hat{F}(N \times I)$ has a $G$-invariant tubular neighbourhood in $M \times I$.

Proof Let us first assume that $F(N \times I) \subset M \backslash \partial M$. By Lemma 8.4, $\widehat{F}(N \times I)$ is a closed smooth neat $G$-invariant submanifold of $(M \backslash \partial M) \times I$. (In fact, one can see, as in the proof of Lemma 8.4, that $\hat{F}(N \times I)$ is closed in $M \times I$.) The claim follows from Theorem 7.3.

If $F(N \times I) \subset \partial M$, then $\widehat{F}(N \times I)$ is a closed smooth neat $G$-invariant submanifold of $\partial M \times I$. By Theorem 7.3, there exists a $G$-invariant tubular neighbourhood $r: U \rightarrow \widehat{F}(N \times I)$ of $\widehat{F}(N \times I)$ in $\partial M \times I$. Let pr: $U \times[0, \infty) \rightarrow U$ be the projection. Then $r \circ$ pr: $U \times[0, \infty) \rightarrow \widehat{F}(N \times I)$ is a $G$-invariant tubular neighbourhood of $\hat{F}(N \times I)=\hat{F}(N \times I) \times\{0\}$ in $\partial M \times I \times[0, \infty)=\partial M \times[0, \infty) \times I$. Using the equivariant collaring theorem yields a $G$-invariant tubular neighbourhood for $\widehat{F}(N \times I)$ in $M \times I$.

Theorem 8.6 Let $G$ be a Lie group and let $M$ be a proper smooth $G$-manifold. Let $N$ be a closed smooth $G$-invariant submanifold of $M$ with $\partial N=\varnothing$. Let $F: N \times I \rightarrow M$ be a $G$-equivariant isotopy having bounded velocity with respect to some complete smooth $G$-invariant Riemannian metric, and such that $F_{0}$ is the canonical inclusion. If either $F(N \times I) \subset \partial M$ or $F(N \times I) \subset M \backslash \partial M$, then $F$ extends to a $G$-equivariant diffeotopy of $M$ which has bounded velocity.

Proof The tangent vectors to the curves

$$
\widehat{F}_{x}: x \times I \rightarrow M \times I, \quad(x, t) \mapsto(F(x, t), t),
$$

define a vector field $Y$ on $\widehat{F}(N \times I)$, where $Y_{(F(x, t), t)}=\left(\frac{\partial F}{\partial t}(x, t), 1\right)$. The horizontal part of $Y$ is a vector field

$$
X: \hat{F}(N \times I) \rightarrow \mathrm{T} M, \quad(y, t) \mapsto X_{(y, t)} \in \mathrm{T}_{y} M .
$$


By Lemma 8.5, there is a $G$-invariant tubular neighbourhood $r: U \rightarrow \widehat{F}(N \times I)$ of $\widehat{F}(N \times I)$ in $M \times I$. Let $f: M \times I \rightarrow[0,1]$ be a smooth $G$-invariant map such that $f \mid \widehat{F}(N \times I)=1$ and $\operatorname{supp}(f) \subset U$. Let $\langle$,$\rangle be a complete smooth G$-invariant Riemannian metric on $M$ such that $F$ has bounded velocity with respect to $\langle$,$\rangle . Let$ $\langle,\rangle^{\prime}$ be a complete smooth $G$-invariant Riemannian metric on $M \times I$. Denote the differential of $r$ at $p \in U$ by $d r_{p}$. Then the restriction $d r_{p} \mid: d r_{p}^{-1}(0)^{\perp} \rightarrow \mathrm{T}_{r(p)} \hat{F}(N \times I)$ is an isomorphism, where $d r_{p}^{-1}(0)^{\perp}$ denotes the orthogonal complement of $d r_{p}^{-1}(0)$ in $\mathrm{T}_{p} U$ and 0 is the origin in $\mathrm{T}_{r(p)} \hat{F}(N \times I)$. Notice that $\frac{\partial F}{\partial t}(x, t)$ lies in $\mathrm{T}_{F(x, t)} M$, for every $(x, t) \in N \times I$, and that $\left(\frac{\partial F}{\partial t}(x, t), 1\right)$ lies in $\mathrm{T}_{(F(x, t), t)} \hat{F}(N \times I)$. Thus $\left(X_{r(p)}, 1\right) \in \mathrm{T}_{r(p)} \hat{F}(N \times I)$. Let pr: TU $\rightarrow \mathrm{T} M$ be the projection. We obtain a smooth invariant vector field

$$
\tilde{X}: U \rightarrow \mathrm{T} M, \quad p \mapsto \operatorname{pr}\left(d r_{p}^{-1}\left(X_{r(p)}, 1\right), 0\right) .
$$

Since $\tilde{X}$ is invariant and the restriction $\tilde{X} \mid \widehat{F}(N \times I)$ has bounded velocity, also $\tilde{X}$ has bounded velocity when $U$ is sufficiently small. Define

$$
\hat{X}: M \times I \rightarrow \mathrm{T} M, \quad(y, t) \mapsto\left\{\begin{aligned}
f(y, t) \tilde{X}(y, t), & \text { if }(y, t) \in U \\
0, & \text { otherwise. }
\end{aligned}\right.
$$

Then $\hat{X}$ is a time-dependent invariant vector field extending $X$. Since $\tilde{X}$ has bounded velocity, it follows that also $\hat{X}$ has bounded velocity. The $G$-equivariant diffeotopy generated by $\hat{X}$ (Theorem 8.1 ) is the required diffeotopy.

Theorem 8.6 implies the following result, which is an equivariant version of Theorem 8.1.5 in [3]:

Theorem 8.7 Let $G$ be a Lie group and let $N$ and $M$ be proper smooth $G$-manifolds. Let $P$ be a closed smooth $G$-invariant submanifold of $N$ such that $P / G$ is compact and $\partial P=\varnothing$. Let $f_{0}, f_{1}: P \rightarrow M \backslash \partial M$ be smooth $G$-equivariant embeddings which are $G$-equivariantly isotopic in $M \backslash \partial M$. If $f_{0}$ extends to a smooth $G$-equivariant embedding $N \rightarrow M$, then so does $f_{1}$.

Proof Let $F: P \times I \rightarrow M \backslash \partial M$ be a $G$-equivariant isotopy such that $F_{0}=f_{0}$ and $F_{1}=f_{1}$. Let id be the identity map of $I$. Then $F \circ\left(f_{0}^{-1} \times\right.$ id $): f_{0}(P) \times I \rightarrow M \backslash \partial M$ is a $G$-equivariant isotopy from the inclusion $i: f_{0}(P) \rightarrow M \backslash \partial M$ to $f_{1} \circ f_{0}^{-1}$. Let $\langle$,$\rangle be a complete smooth G$-invariant Riemannian metric on $M$. Since $f_{0}(P) / G$ is compact, it follows that the isotopy $F \circ\left(f_{0}^{-1} \times\right.$ id $)$ has bounded velocity. It now follows from Theorem 8.6, that $F \circ\left(f_{0}^{-1} \times\right.$ id $)$ extends to a $G$-equivariant diffeotopy $H$ of $M$. Thus $H_{1}: M \rightarrow M$ is a smooth $G$-equivariant diffeomorphism such that the restriction $H_{1} \mid f_{0}(P)=f_{1} \circ f_{0}^{-1}$. Therefore, if $h: N \rightarrow M$ is a smooth $G$-equivariant 
embedding extending $f_{0}$, then $H_{1} \circ h$ is a smooth $G$-equivariant embedding extending $f_{1}$.

\section{A $G$-equivariant smoothing theorem}

Let $G$ be a Lie group and let $M$ be a proper smooth $G$-manifold with boundary $\partial M$. Let $f_{i}: \partial M \times[0, \infty) \rightarrow U_{i} \subset M, i=0,1$, be $G$-equivariant collars of $\partial M$.

Definition 9.1 The collars $f_{0}$ and $f_{1}$ are said to have $G$-equivariantly isotopic $\partial M-$ germs, if there exist an open $G$-invariant neighbourhood $V$ of $\partial M$ in $U_{0} \cap U_{1}$ and a $G$-equivariant isotopy $F: V \times I \rightarrow M$ such that

- $F_{t}(x)=x$, for every $x \in \partial M$,

- $F_{0}$ equals the inclusion $i: V \rightarrow M$,

- $F_{1}=f_{1} \circ f_{0}^{-1} \mid V$,

- $\hat{F}(V \times I)$ is open in $M \times I$.

Theorem 9.2 Let $G$ be a Lie group and let $M$ be a proper smooth $G$-manifold with boundary $\partial M$. Then any two $G$-equivariant collars of $\partial M$ in $M$ have $G-$ equivariantly isotopic $\partial M$-germs.

Proof Let $f_{i}: \partial M \times[0, \infty) \rightarrow U_{i} \subset M, i=1,2$, be $G$-equivariant collars of $M$. It follows easily, by applying Theorem 4.6, that there exists a $G$-equivariant rel $\partial M$ isotopy $F: \partial M \times[0, \infty) \times I \rightarrow M$ satisfying the conditions of Definition 4.5 for these collars. Let $V=U_{0} \cap U_{1}$. Then $F \circ\left(f_{0}^{-1} \mid V \times \mathrm{id}_{I}\right): V \times I \rightarrow M$ is a $G$-equivariant rel $\partial M$ isotopy satisfying the first two conditions and the last condition of Definition 9.1. It is left for the reader to find a $G$-equivariant map $H: \partial M \times[0, \infty) \times I \rightarrow \partial M \times[0, \infty) \times I$ such that $F \circ H \circ\left(f_{0}^{-1} \mid V \times \operatorname{id}_{I}\right)$ satisfies all the conditions of Definition 9.1.

Theorem 9.3 Let $G$ be a Lie group and let $M$ be a proper smooth $G$-manifold with boundary $\partial M$. Let $U$ be an open $G$-invariant neighbourhood of $\partial M$ in $M$. Let $F$ be a $G$-equivariant isotopy of $\partial M$-germs of two $G$-equivariant collars of $\partial M$. Then there exists a $G$-equivariant diffeotopy $\widetilde{F}$ of $M$ having support in $U$ and such that $\tilde{F}|(V \times I)=F|(V \times I)$, for some $G$-invariant neighbourhood $V$ of $\partial M$ in $M$. Moreover, $\widetilde{F}_{0}$ equals the identity map of $M$.

Proof Let $\langle\rangle:, \mathrm{T} M \oplus \mathrm{T} M \rightarrow \mathbb{R}$ be a complete smooth $G$-invariant Riemannian metric of $M$. The $G$-equivariant isotopy $F$ is defined on $U_{0} \times I$, where $U_{0}$ is some 
$G$-invariant neighbourhood of $\partial M$ in $M$. We may assume that $F\left(U_{0} \times I\right) \subset U$. The isotopy $F$ keeps $\partial M$ pointwise fixed. Consequently, $F$ has bounded velocity in some $G$-invariant neighbourhood $V_{0}$ of $\partial M$. By Theorem 8.3, there exists a $G$-diffeotopy $\widetilde{F}$ of $M$, which agrees with $F$ on $V \times I$, where $V \subset V_{0}$ is some $G$-invariant neighbourhood of $\partial M$, whose support is in $F\left(V_{0} \times I\right) \subset U$ and such that $\widetilde{F}_{0}=\operatorname{id}_{M}$.

Let $G$ be a Lie group and let $M$ and $N$ be proper smooth $G$-manifolds. Let $A_{0}$ and $A_{1}$ be $G$-invariant subsets of $M$ and let $M=A_{0} \cup A_{1}$. Assume $f_{i}: A_{i} \rightarrow N$, $i=0,1$, are $G$-equivariant maps such that $f_{0}(x)=f_{1}(x)$, for every $x \in A_{0} \cap A_{1}$. We then define the $G$-equivariant map $f_{0} \cup f_{1}: M \rightarrow N$ by $\left(f_{0} \cup f_{1}\right)(x)=f_{0}(x)$ when $x \in A_{0}$ and $\left(f_{0} \cup f_{1}\right)(x)=f_{1}(x)$ when $x \in A_{1}$.

The following result is an equivariant version of Theorem 8.1.9 in [3]:

Theorem 9.4 Let $G$ be a Lie group. For $i=0,1$, let $W_{i}$ be a proper smooth $G-$ manifold with dimension $n$ and without boundary. Assume that each $W_{i}$ is the union of two closed n-dimensional $G$-invariant submanifolds $M_{i}$ and $N_{i}$ such that

$$
M_{i} \cap N_{i}=\partial M_{i}=\partial N_{i}=V_{i} .
$$

Let $h: W_{0} \rightarrow W_{1}$ be a $G$-equivariant homeomorphism which maps $M_{0}$ and $N_{0}$ diffeomorphically onto $M_{1}$ and $N_{1}$, respectively. Then there exists a $G$-equivariant diffeomorphism $f: W_{0} \rightarrow W_{1}$ such that we have $f\left(M_{0}\right)=M_{1}, f\left(N_{0}\right)=N_{1}$ and $f\left|V_{0}=h\right| V_{0}$. Moreover, $f$ can be chosen to coincide with $h$ outside a given $G-$ invariant neighbourhood $Q$ of $V_{0}$.

Proof By Theorem 4.4, there exist $G$-invariant tubular neighbourhoods $\tau_{i}$ of $V_{i}$ in $W_{i}, i=0,1$. Then $\tau_{0}$ defines a $G$-equivariant collar $f_{0}: V_{0} \times[0, \infty) \rightarrow M_{0}$, denoted by $\tau_{0} \mid M_{0}$. Similarly, we denote the $G$-equivariant collar $f_{1}: V_{1} \times[0, \infty) \rightarrow M_{1}$ by $\tau_{1} \mid M_{1}$. This collar then induces another $G$-equivariant collar for $V_{0}$ in $M_{0}$,

$$
h^{-1} \circ f_{1} \circ(h \times \mathrm{id}): V_{0} \times[0, \infty) \rightarrow M_{0},
$$

which we denote by $h^{-1}\left(\tau_{1} \mid M_{1}\right)$.

By Theorem 9.2, the collars $\tau_{0} \mid M_{0}$ and $h^{-1}\left(\tau_{1} \mid M_{1}\right)$ have $G$-equivariantly isotopic $\partial M$-germs, ie there exists a $G$-equivariant isotopy $H: U_{0} \times I \rightarrow M_{0}$ such that $H_{t}(x)=x$, for every $x \in V_{0}, H_{0}$ equals the inclusion $i: U_{0} \rightarrow M_{0}, H_{1}$ is the composition $h^{-1} \circ f_{1} \circ(h \times \mathrm{id}) \circ f_{0}^{-1} \mid U_{0}$ and $\hat{H}\left(U_{0} \times I\right)$ is open in $M_{0} \times I$. Here,

$$
U_{0} \subset f_{0}\left(V_{0} \times[0, \infty)\right) \cap h^{-1} \circ f_{1} \circ(h \times \mathrm{id})\left(V_{0} \times[0, \infty)\right)
$$


is an open $G$-invariant neighbourhood of $V_{0}$ in $M_{0}$. By Theorem 9.3, there exists a $G$-equivariant diffeotopy $\widetilde{H}$ of $M_{0}$ having support in $M_{0} \cap Q$ and such that $\tilde{H}|(V \times I)=H|(V \times I)$, for some $G$-invariant neighbourhood $V$ of $V_{0}$ in $M_{0}$ and $\widetilde{H}_{0}=\operatorname{id}_{M_{0}}$. But then $\widetilde{F}=h \circ \widetilde{H}: M_{0} \times I \rightarrow M_{1}$ is a $G$-equivariant isotopy, $\widetilde{F}_{0}=h\left|M_{0}, \widetilde{F}_{1}\right| V_{0}=h \mid V_{0}$ and $\widetilde{F}_{1}\left|\left(M_{0} \backslash Q\right)=h\right|\left(M_{0} \backslash Q\right)$. Moreover, $\widetilde{F}_{1}\left(\tau_{0} \mid M_{0}\right)$ and $\tau_{1} \mid M_{1}$ have the same $V_{1}$-germs, ie there exists a neighbourhood $V^{\prime}$ of $V_{1}$ in $V_{1} \times[0, \infty)$ such that $\widetilde{F}_{1} \circ f_{0} \circ\left(h^{-1} \times\right.$ id $)\left|V^{\prime}=f_{1}\right| V^{\prime}$. We denote $\widetilde{F}_{1}$ by $f^{\prime}$.

Similarly, we can isotop $h \mid N_{0}: N_{0} \rightarrow N_{1}$ equivariantly to a $G$-equivariant diffeomorphism $f^{\prime \prime}: N_{0} \rightarrow N_{1}$ such that $f^{\prime \prime}$ equals $h$ on $V_{0}$ and on $N_{0} \backslash Q$ and the collar $f^{\prime \prime}\left(\tau_{0} \mid N_{0}\right)$ has the same $V_{1}$-germ as $\tau_{1} \mid N_{1}$. The map $f=f^{\prime} \cup f^{\prime \prime}: W_{0} \rightarrow W_{1}$ is the required $G$-equivariant diffeomorphism.

Remark 9.5 Using Corollary 6.3 and Proposition 4.3, it is possible to choose the tubular neighbourhood $\tau_{1}$ in such a way that the collars $\tau_{1} \mid M_{1}$ and $h\left(\tau_{0} \mid M_{0}\right)$ have the same $V_{1}$-germs. Thus we can make $f$ to equal $h$ on $M_{0}$ (or on $N_{0}$ ).

\section{Equivariant gluing}

Let $G$ be a Lie group and let $M$ and $N$ be proper smooth $G$-manifolds with boundary. Assume $f: \partial M \rightarrow \partial N$ is a $G$-equivariant diffeomorphism. Then the adjunction space $W=M \cup_{f} N$ is a topological manifold on which $G$ acts properly and continuously.

We identify $M$ and $N$ with their images in $W$. Let $\partial M=\partial N=V$. Using equivariant collars of $V$ in $M$ and $N$, we obtain a $G$-equivariant homeomorphism of a $G-$ invariant neighbourhood $U$ of $V$ in $W$ onto $V \times \mathbb{R}$ which takes $x \in V$ to $(x, 0)$, and which maps $U \cap M$ and $U \cap N$ diffeomorphically onto $V \times[0, \infty)$ and $V \times(-\infty, 0]$, respectively. This homeomorphism induces a differential structure on $U$. Collation of differential structures [3, p 13] of $U, M$ and $N$ gives a differential structure for $W$. By Theorem 9.4, the $G$-diffeomorphism type of $W$ obtained by equivariant gluing is unique. Using Remark 9.5, we obtain the following result (an equivariant version of Theorem 8.2.1 in [3]):

Theorem 10.1 Let $G$ be a Lie group and let $M$ and $N$ be proper smooth $G-$ manifolds with boundary. Let $f: \partial M \rightarrow \partial N$ be a $G$-equivariant diffeomorphism. Let $\alpha$ and $\beta$ be two differential structures on $W=M \cup_{f} N$ which both induce the original structures on $M$ and $N$. Then there is a $G$-equivariant diffeomorphism $h: W_{\alpha} \rightarrow W_{\beta}$ such that $h \mid M=\mathrm{id}_{M}$.

The following is an equivariant version of Theorem 8.2.2 in [3]: 
Theorem 10.2 Let $G$ be a Lie group and let $M_{0}, M_{1}$ and $N$ be proper smooth $G$-manifolds with boundary. Let $f_{i}: \partial M_{i} \rightarrow \partial N, i=0,1$, be $G$-equivariant diffeomorphisms. Suppose that the $G$-equivariant diffeomorphism $f_{1}^{-1} \circ f_{0}: \partial M_{0} \rightarrow \partial M_{1}$ extends to a $G$-equivariant diffeomorphism $h: M_{0} \rightarrow M_{1}$. Then $M_{0} \cup_{f_{0}} N \approx_{G}$ $M_{1} \cup_{f_{1}} N$.

Proof Define the map between adjuction spaces

$$
\psi: M_{0} \cup_{f_{0}} N \rightarrow M_{1} \cup_{f_{1}} N
$$

by $\psi \mid M_{0}=h$ and $\psi \mid N=\mathrm{id}_{N}$. Then $\psi$ is a $G$-equivariant homeomorphism mapping $M_{0}$ and $N$ diffeomorphically onto $M_{1}$ and $N$, respectively. The claim now follows from Theorem 9.4.

The following result is an equivariant version of Theorem 8.2.3 in [3]:

Theorem 10.3 Let $G$ be a Lie group and let $M$ and $N$ be proper smooth $G-$ manifolds with boundary. Let $f_{i}: \partial M \rightarrow \partial N, i=0,1$, be $G$-equivariantly isotopic $G$-equivariant diffeomorphisms. Then $M \cup_{f_{0}} N \approx_{G} M \cup_{f_{1}} N$.

Proof Let $F: \partial M \times I \rightarrow \partial N$ be a $G$-equivariant isotopy with $F_{0}=f_{0}$ and $F_{1}=f_{1}$. We may assume that $F$ is a constant isotopy near 1 . Then $H=f_{1}^{-1} \circ F: \partial M \times I \rightarrow \partial M$ is a $G$-equivariant isotopy with $H_{0}=f_{1}^{-1} \circ f_{0}$ and $H_{t}=H_{1}=\mathrm{id}_{\partial M}$, when $t$ is near 1 . By the equivariant collaring theorem, we can identify $\partial M \times I$ with a $G-$ invariant neighbourhood $O$ of $\partial M$ in $M$. The map $\hat{H}: \partial M \times I \rightarrow \partial M \times I$ defines a $G$-equivariant diffeomorphism $h: O \rightarrow h(O)$. Let id be the identity map of $M \backslash O$. Then $h \cup$ id: $M=O \cup(M \backslash O) \rightarrow h(O) \cup(M \backslash O)$ is a $G$-equivariant diffeomorphism extending $f_{1}^{-1} \circ f_{0}$. By Theorem 10.2, $M \cup_{f_{0}} N \approx_{G}(h(O) \cup(M \backslash O)) \cup_{f_{1}} N$. The claim follows, since $(h(O) \cup(M \backslash O)) \cup_{f_{1}} N \approx_{G} M \cup_{f_{1}} N$.

\section{References}

[1] G E Bredon, Introduction to compact transformation groups, Pure and Applied Mathematics 46, Academic Press, New York (1972) MR0413144

[2] M Brown, Locally flat imbeddings of topological manifolds, Ann. of Math. (2) 75 (1962) 331-341 MR0133812

[3] M W Hirsch, Differential topology, Graduate Texts in Mathematics 33, Springer, New York (1976) MR0448362

[4] S Illman, M Kankaanrinta, A new topology for the set $C^{\infty, G}(M, N)$ of $G-$ equivariant smooth maps, Math. Ann. 316 (2000) 139-168 MR1735082 
[5] S Illman, M Kankaanrinta, Three basic results for real analytic proper $G$-manifolds, Math. Ann. 316 (2000) 169-183 MR1735083

[6] M Kankaanrinta, Proper smooth $G$-manifolds have complete $G$-invariant Riemannian metrics, Topology Appl. 153 (2005) 610-619 MR2193330

[7] JL Koszul, Sur certains groupes de transformations de Lie, from: "Géométrie différentielle. Colloques Internationaux du Centre National de la Recherche Scientifique, Strasbourg, 1953”, Centre National de la Recherche Scientifique, Paris (1953) 137-141 MR0059919

[8] S Lang, Differential and Riemannian manifolds, third edition, Graduate Texts in Mathematics 160, Springer, New York (1995) MR1335233

[9] J N Mather, Stability of $C^{\infty}$ mappings. II. Infinitesimal stability implies stability, Ann. of Math. (2) 89 (1969) 254-291 MR0259953

[10] G D Mostow, Equivariant embeddings in Euclidean space, Ann. of Math. (2) 65 (1957) 432-446 MR0087037

[11] R S Palais, Imbedding of compact, differentiable transformation groups in orthogonal representations, J. Math. Mech. 6 (1957) 673-678 MR0092927

[12] RS Palais, The classification of $G$-spaces, Mem. Amer. Math. Soc. No. 36 (1960) MR0177401

[13] R S Palais, On the existence of slices for actions of non-compact Lie groups, Ann. of Math. (2) 73 (1961) 295-323 MR0126506

[14] M J Pflaum, Analytic and geometric study of stratified spaces, Lecture Notes in Mathematics 1768, Springer, Berlin (2001) MR1869601

[15] G W Schwarz, Lifting smooth homotopies of orbit spaces, Inst. Hautes Études Sci. Publ. Math. (1980) 37-135 MR573821

Department of Mathematics, PO Box 400137, University of Virginia

Charlottesville VA 22904-4137, USA

mk5aq@virginia.edu

Received: 19 January 2006 Revised: 30 October 2006 